\title{
La mémoire de l'homme
}

\author{
Claudine Potvin
}

\begin{abstract}
Man's memory
The girl knew she had to remember the text hit the SAVE key but the mother's body effaced itself. Later on drunk with false memories she will think she's lost her... Daily she prepared a detailed list of childhood memories in a large book. One night she found the portrait of two bodies on the shore the touch of lips blocked out by a fly on the lens of the camera incessantly interrupts the breathing of the girl sprawled on the wet pebbles like an obsession she cuts the negative the man's hands plunge into the cleavage of her bathing suit. Because of him in time this book of memoires became a pornographic album. Pages went missing. A girl's memory is painful; women tend not to remember much.... She could never remember the end of a film. She refused the final scene, the closure, just as she denied history. Return was nothing but a delirious projection. Did one have to enlarge the screen to project the abuse the rejection the violence? Only in order to remember that one shouldn't try it. Amnesiacs, her sisters leafed through women's texts hoping to rediscover vanished chronicles. They invented tales full of realisms a real without traps. She watched them repeated that she too knew how to replace all the details all the forgotten things one by one... and she closed the covers ripped the sheets destroyed the film. Man's memory too she effaced in this way. Now she remembered.
\end{abstract}

L'instant où la mère ne la touchait plus ne bordait plus son sourire d'enfant était resté figé dans sa mémoire elle préférait se le remémorer à l'aube car elle n'existait presque plus coincée sans doute entre l'absence de l'homme et le dissentiment. A la limite d'un bijou finement ciselé un bracelet mexicain plus tard elle croira l'avoir perdu retrouver une mère inutile ivre de faux souvenirs. L'argent ternit encore la lueur de sa peau grise sous la cendre. 
La fille savait qu'il fallait garder le texte en mémoire appuyer sur la case save mais le corps de la mère s'effaçait de lui-même les pleurs des autres n'y changeant rien.

Un jour elle rejouerait cette scène à l'infini sur le mode grotesque et elles en rieraient ensemble coupées de leurs mémoires ces femmes du temps jadis s'écrivent des lettres d'amour se racontent un moyen âge une courtoisie oubliée des songes enfermés dans les tourelles du vent l'amant ne vient plus y lire à haute voix le chant de l'aigle.

Elle ne se rappellait jamais la fin du film. Pour elle, le cinéma signifiait dans le processus même du déroulement et dans le cumul des images que l'on veut bien garder à la fin dans un coffre d'étain ou de bois où s'accumulait une forme du passé. Elle refusait la scène finale la clôture tout comme elle niait l'histoire. La répétition n'existait donc pas et le retour n'avait de sens que dans une projection délirante de son univers, une fuite statique en fait.

Les femmes ne se souviennent pas de grand chose si l'on entend par là les hauts faits de l'histoire les évènements marquants les changements de gouvernements la fille disait toujours que ce n'était pas vrai qu'elles savaient tout ce que les hommes ignorent et tout ce qu'on énumérait dans les journaux et qui ne concernait personne surtout pas les femmes mais qu'elles avaient dû choisir au moyen âge précisément entre le souvenir des croisades l'ironie des princes le froid des châteaux la difficulté d'aimer et la mémoire intérieure. Elles avaient préféré l'âme et le coeur bien sûr et construisent depuis ce jour des catalogues de souvenirs intimes. La fille se fabriquait des inventaires photographiques selon l'humeur amnésique de ses désirs.

Elle se souvenait de ce conte de Borges de la mémoire infaillible de Funes el memorioso de l'homme qui reconstruisait ses rêves elle préparait quotidiennement dans un grand cahier à la couverture mauve une liste 
détaillée de ses souvenirs d'enfance d'aussi loin qu'elle se souvienne chaque jour tentait de remonter un peu plus loin.

Une nuit elle avait ainsi retrouvé le portrait de deux corps sur la grève figés dans le sable l'échange des lèvres bloqué par le vol d'une mouche sur la lentille la caméra interrompt sans cesse le souffle de la fille étendue sur les pierres mouillées comme une obsession elle découpe le négatif les mains de l'homme plongeant dans l'échancrure de son maillot. A cause de lui le cahier des souvenirs devenait avec le temps un album pornographique. Des pages s'y absentaient des années y refusaient le temps. La mémoire des filles est douloureuse.

La pensée des femmes s'inscrivait sur sa peau traces de possessions marques d'accouchements viols intimes les rides effaçant peu à peu l'âge du chagrin les lignes du corps se repliant sur le coeur et l'émotion. Fallait-il revoir le film qui menait toujours à la même équivoque? Chaque équivoque, chaque malentendu suscite la mort. Fallait-il agrandir l'écran et y projeter le regret, le refus, l'abus, l'excès, la violence, le noir? Pour se rappeler qu'elle n'aurait pas dû.

La pluie inondait parfois le balcon dégringolait l'escalier tel un rire s'insérait entre les doigts des enfants elle regardait le spectacle de la mère curieuse elle dirigeait les jumelles de l'autre côté de l'étang où jouaient les garçons sa langue se baladait sur le rebord de la balustrade le désir blanc du marbre glacé effleurant le métal et le cuir de l'objet qu'elle serrait sur sa poitrine.

Elle aimait cette sensation de l'eau qui coulait abondamment entre ses cuisses d'adolescente elle imaginait toujours voir sa mère pleurer pendant des heures et le père approcher le vase de porcelaine pour conserver les larmes comme les gouttes de pluie pour laver la chevelure des filles. La tentation de l'eau le corps du désert le mouvement du soleil autour de l'oasis se raccrochaient aux palmiers au vent sec de la prairie à l'existence de la mémoire à la foire à la figure maternelle.

Amnésiques, toutes ses soeurs recopiaient tous les mots féminins de tous les livres de femmes pour retrouver l'odeur de l'enfance recréer la profondeur du sexe la fluidité du plaisir les parois humides le nom la couleur de la faune et de la flore les espaces entre la chair rose et les 
robes d'été. La configuration des mots leur révélait des géographies ignorées de la mère des chroniques disparues.

Elles se promenaient dans le langage à la manière des belles folles du cinéma muet racontant à qui voulait les entendre que le corps est la seule souvenance des femmes, le miroir des mystiques, l'avenir, l'attente secrète. Elles dansaient d'une ligne à l'autre soulignaient la possibilité d'un passé commentaient les confidences des écrivaines s'inventaient des histoires une vie de pauses de retours et de bonds des récits pleins de réalismes un réel sans pièges.

Elle les observait s'amusait de leurs jeux répétait que le passé ne s'apprivoise pas elle savait elle copier le temps remonter le cours des menstrues saisonnières aligner les hommes de leur jeunesse replacer un à un tous les détails tous les oublis elle se voulait absolue le repli de la mémoire s'installait à la naissance de l'épaule support de gestes anciens que la mère ignorait le baiser la caresse le toucher elle aimait se souvenir du regard des filles accroché à l'océan retenir les filets des heures allongées l'écume salée des ivrognes.

Puis elle refermait le couvercle déchirait les feuilles détruisait le film disséquant les visages le tableau en pièces détachées une oreille traîne dans le décor un oeil s'échappe une bouche rouge sang déteint sur la nappe blanche elle ramassait la coupe renversée.

Elle effaçait toujours la mémoire de l'homme de la même manière. Elle s'en souvenait maintenant. 